\title{
Analyse Granulometrique et Cartographie des Sediments Superficiels de la Lagune Cotiere au Benin (Afrique de L'Ouest)
}

\section{Tossou Edjrogan Michaël, PhD Student}

Laboratoire de Géologie Mines \& Environnement, Faculté des Sciences et Techniques, Université d'Abomey-Calavi, Bénin. Laboratoire Leïdi, Dynamiques des territoires et développement, Université Gaston Berger de

Saint-Louis, Sénégal. Laboratoire d'Enseignement et de Recherche en Géomatique, Ecole Supérieure Polytechnique, Université Cheikh Anta Diop de Dakar, Sénégal. Chaire Internationale en Physique Mathématique et Applications/UNESCO, Faculté des Sciences et Techniques, Université d'Abomey-Calavi, Bénin

Dr. Adechina Archange Rodrigue, Dr. Hounkpe Bidossèssi Jéchonias, Prof. Kelome Carine Nelly,

Laboratoire de Géologie Mines \& Environnement, Faculté des Sciences et Techniques, Université d'Abomey-Calavi, Bénin Prof. Sy Boubou Aldiouma,

Laboratoire Leïdi, Dynamiques des territoires et développement, Université Gaston Berger de Saint-Louis, Sénégal

\section{Prof. Diaw Amadou Tahirou,}

Laboratoire d'Enseignement et de Recherche en Géomatique, Ecole Supérieure Polytechnique, Université Cheikh Anta Diop de Dakar, Sénégal

Doi:10.19044/esj.2019.v15n24p294 URL:http://dx.doi.org/10.19044/esj.2019.v15n24p294

\section{Résumé}

Cette étude s'inscrit dans la problématique de la dynamique sédimentaire des hydrosystèmes paraliques. Elle se base sur les techniques granulométriques pour caractériser les sédiments de la lagune côtière, l'un des quatre principaux plans d'eau paraliques du Bénin. A partir des prélèvements de sédiments superficiels sur 48 transects, des travaux de laboratoire, des calculs de paramètres texturaux de Folk \& Ward sur Excel et le logiciel Origin 6.0 ainsi que la cartographie des faciès via ArcGIS 10.3., ont été effectués. Les indices granulométriques de ces faciès indiquent un classement médiocre de sables moyens, presque symétrique, avec une acuité mésocurtique. Le diagramme de Passega décrit un transport par saltation ou roulement de ces 
sables. La combinaison des diagrammes de Friedman, Moiola et Weiser montre des sédiments d'origine fluviatile et ceux remobilisés à partir des dunes côtières adjacentes à la lagune. Un zonage hydrodynamique est également noté, caractérisé par un hydrodynamisme faible du secteur oriental ou lagune de Ouidah et un hydrodynamisme relativement fort du secteur occidental ou lagune de Grand-Popo, avec une vitesse de l'ordre de 0,78 à 1,4 $\mathrm{m} / \mathrm{s}$ vers le fond et un débit de $429 \mathrm{~m}^{3} / \mathrm{s}$, à la station de Avlo Plage. En définitive, ce papier fait ressortir l'état de variations hydrodynamiques de la lagune, notamment une opposition entre secteurs oriental et occidental, la diversité des sources d'apports sédimentaires et la réalisation de la première carte des lithofaciès de cet environnement aquatique, outil indispensable à son suivi face à des changements futurs et à un contexte en mutation perpétuelle.

Mots-clés : Lagune côtière, Dynamique sédimentaire, Granulométrie, Paramètres texturaux, ArcGIS 


\title{
Grain-size Analysis and Shallow Sediments Mapping of the Benin Coastal Lagoon (West Africa)
}

\begin{abstract}
Tossou Edjrogan Michaël, PhD Student
Laboratoire de Géologie Mines \& Environnement, Faculté des Sciences et Techniques, Université d'Abomey-Calavi, Bénin. Laboratoire Leïdi, Dynamiques des territoires et développement, Université Gaston Berger de Saint-Louis, Sénégal. Laboratoire d'Enseignement et de Recherche en Géomatique, Ecole Supérieure Polytechnique, Université Cheikh Anta Diop de Dakar, Sénégal. Chaire Internationale en Physique Mathématique et Applications/UNESCO, Faculté des Sciences et Techniques, Université d'Abomey-Calavi, Bénin

Dr. Adechina Archange Rodrigue, Dr. Hounkpe Bidossèssi Jéchonias, Prof. Kelome Carine Nelly,

Laboratoire de Géologie Mines \& Environnement, Faculté des Sciences et Techniques, Université d'Abomey-Calavi, Bénin
\end{abstract}

Prof. Sy Boubou Aldiouma,

Laboratoire Leïdi, Dynamiques des territoires et développement,

Université Gaston Berger de Saint-Louis, Sénégal

Prof. Diaw Amadou Tahirou,

Laboratoire d'Enseignement et de Recherche en Géomatique, Ecole

Supérieure Polytechnique, Université Cheikh Anta Diop de Dakar, Sénégal

Abstract

The current study is part of the wide-ranging issue related to the sedimentary dynamics of paralic hydro systems. This study aims to characterize the sedimentary cover of the coastal lagoon, one of Benin's four main water bodies, using the granulometric analysis procedure. Based on shallow sediment samples', following 48 transects in the lagoon, a few laboratory processes have been undertaken, including granulometry, statistical calculus acting upon the Folk \& Ward textural parameters through Excel and Origin 6.0 software, and also sandy feature mapping, while considering the ArcGIS 10.3 software performance. The grain size indexes of these facies indicate a second or poor-rate classification of medium sands, almost symmetrical, with mesokurtic acuity. The Passega diagram describes a saltation or rolling transport of these sands. The combination of Friedman's, 
Moiola's and Weiser's diagrams reveals fluvial-origin sediments and remobilization from the coastal dune zones flanking the lagoon. At the level of the coastal lagoon, a hydrodynamic zoning is established, characterized by some insubstantial hydro dynamism of the eastern sector, impacted by the influence of the so-called Ouidah lagoon and a relatively strong hydro dynamism of the western sector corresponding to the Grand- Popo lagoon, with a speed ranging between 0.78 to $1.4 \mathrm{~m} / \mathrm{s}$ towards the bottom and a flow rate of $429 \mathrm{~m}^{3} / \mathrm{s}$, at the Avlo beach station. Ultimately, this study made it possible to underscore the state of hydrodynamic variations observed in the lagoon, notably an opposition between eastern and western sectors, the diversity of sedimentary input sources and the design of the first lithological facies map of this paralic environment, which now stands as a vital tool for its follow-up and monitoring, in the current context of upcoming changes and unremitting alteration.

Keywords: Coastal Lagoon, Sedimentary Dynamics, Granulometry, Textural parameters, ArcGIS

\section{Introduction}

Les lagunes sont souvent des milieux éphémères et relativement stables même à l'état naturel. Leurs organisations et caractéristiques sont étroitement liées à un équilibre dynamique entre les apports marins et continentaux (Maanan, 2003). Les tempêtes, les submersions marines, les aménagements dans les bassins versants et les tributaires de ces plans d'eau, auxquels se surimpose parfois la dynamique des embouchures, peuvent rapidement rompre cet équilibre et altérer ainsi leurs richesses. Selon Castaings (2008), les lagunes représentent un atout exceptionnel pour les activités économiques traditionnelles, les nouvelles formes de tourisme et la perpétuation d'un milieu riche et vivant. Avec les autres types de zones humides du domaine côtier, elles représentent un patrimoine écologique exceptionnel dont la richesse de la biodiversité est reconnue internationalement par la convention de Ramsar depuis 1971. Ainsi, il est d'un intérêt certain de comprendre et de tenter d'expliquer les processus hydrodynamiques et sédimentologiques de ces milieux afin de mieux les gérer (N'guessan et al., 2014). Au Bénin, si les niveaux de connaissances scientifiques de certains plans d'eau sont déjà élaborés et font l'objet de suivi de la dynamique sédimentaire, la lagune côtière, l'un des (4) quatre principaux plans d'eau côtiers, demeure mal connue quant à la caractérisation récente de sa couverture sédimentaire et la répartition de ses faciès. Malgré les migrations profondes et la fermeture de l'embouchure dénommée la Bouche du Roi, suivies par des ouvertures mécaniques périodiques (Laibi, 2011), aucune étude sédimentologique et morphologique n'a été entreprise sur toute l'étendue de 
la lagune côtière (de Grand-Popo à Togbin). Or, la compréhension du fonctionnement hydrodynamique et sédimentaire des lagunes constitue un élément prépondérant pour leur aménagement et une base d'évaluation des changements de leur riche biodiversité. Aussi, à travers une approche sédimentologique fondée sur une analyse granulométrique, ce papier précise la nature, l'origine, le mode de transport des sédiments tapissant le fond de la lagune et établit la carte de répartition spatiale de ses différents faciès.

\section{Présentation de la zone d'étude}

La lagune côtière est un plan d'eau intercommunal, située dans la partie méridionale du Bénin, entre les parallèles $6^{\circ} 16^{\prime}$ et $6^{\circ} 23^{\prime}$ de latitude Nord et les méridiens $1^{\circ} 50^{\prime}$ et $2^{\circ} 22$ de longitude Est (Figure 1). A l'Ouest, sur environ $15 \mathrm{~km}$ d'extension, elle porte le nom de lagune de Grand-Popo et à l'Est, sur $40 \mathrm{~km}$, plus précisément après le débouché du chenal Ahô dans la lagune jusqu'à hauteur de la localité de Togbin, elle est désignée sous le vocable de lagune de Ouidah. Elle couvre une superficie d'environ $30 \mathrm{~km}^{2}$ et fait partie intégrante du site Ramsar $n^{\circ} 1017$.Son régime hydrodynamique est marqué par l'océan Atlantique et les cours d'eau tels que le fleuve Mono, la rivière Sazoué et le fleuve Couffo dont les eaux séjournent dans le "lac" Ahémé pour transiter vers la lagune via le chenal Ahô. Le climat régnant sur la zone d'étude est de type subéquatorial avec une pluviométrie annuelle moyenne de $1270 \mathrm{~mm}$ sur une chronique de 1970-2016. Au plan géomorphologique, la lagune côtière est située dans une plaine littorale, constituée de cordons sableux d'âges différents, dont les plus récents, dits cordons subactuels de sable brun, d'altitude comprise entre 1 à $3 \mathrm{~m}$ et larges de 100 à $300 \mathrm{~m}$, la protègent des assauts de l'océan Atlantique. La communication océan-lagune s'établit dans partie occidentale via l'embouchure de la Bouche du Roi. Les activités telles que la pêche, l'agriculture, la saliculture, le tourisme, le commerce y sont développées. Compte-tenu de son étroitesse et de sa longueur de l'ordre de $55 \mathrm{~km}$, la zone d'étude a été sectionnée en trois (3) portions ( $\mathrm{a}, \mathrm{b}$ et $\mathrm{c})$, de manière à accroitre autant que possible la résolution de l'image de la lagune côtière. 


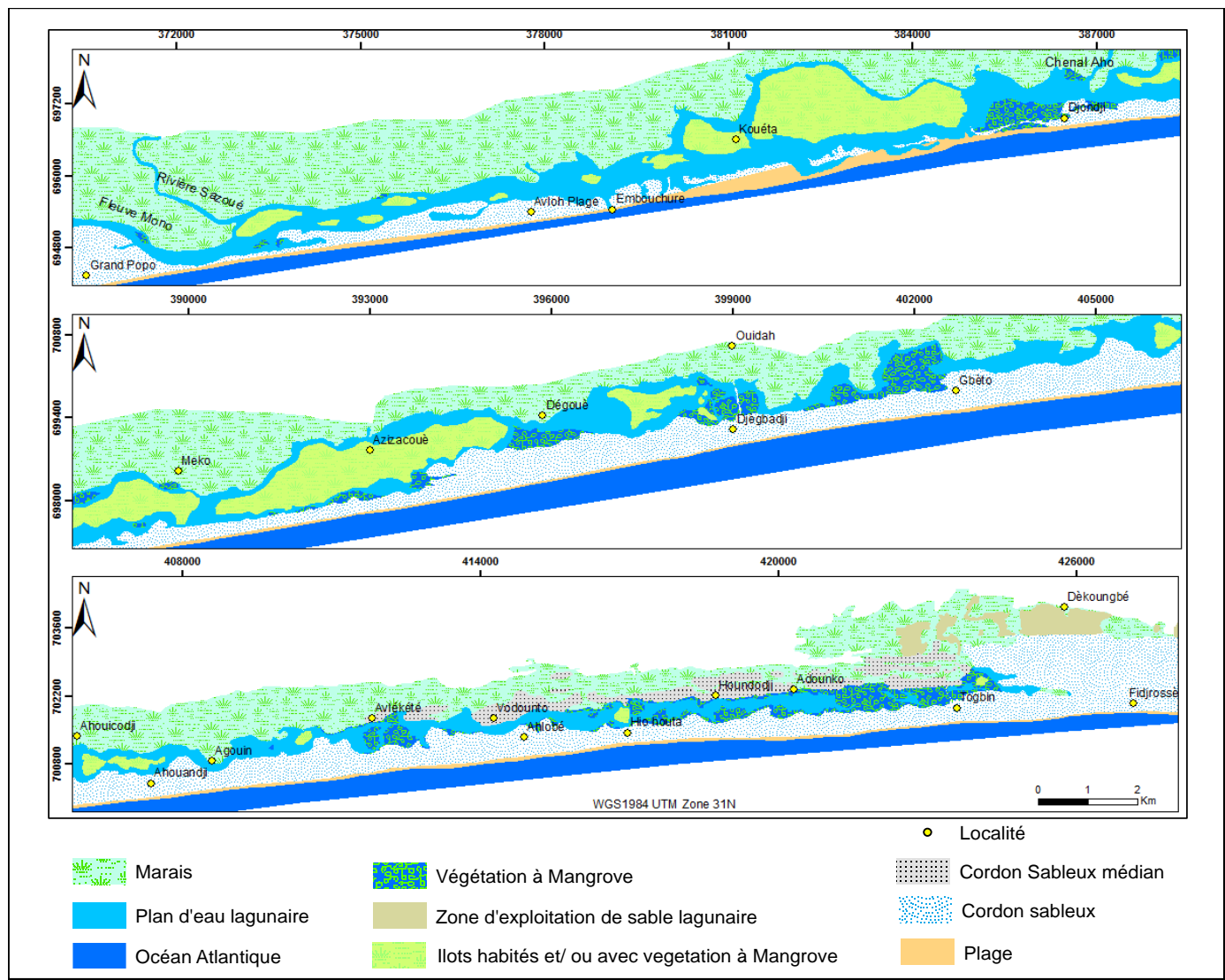

Fig. 1. Carte de présentation de la zone d'étude, découpée en 3 secteurs d'Ouest en Est a-) Partie Ouest : de la confluence du Mono et de la Sazoué au voisinage de Méko après le chenal Aho ;

b-) Partie centrale : du village de Méko à Ahouicodji ;

c-) Partie Est : du village de Ahouicodji à Togbin.

\section{Matériel et méthodes}

\subsection{Echantillonnage}

Des campagnes de prélèvements de sédiments superficiels ont été effectuées en juin 2017. Le matériel déployé comprend: une barque motorisée, une benne, un GPS GARMIN, des sachets, des marqueurs permanents et un appareil photo numérique. Au total, 200 échantillons de sédiments ont été prélevés entre 5 et $15 \mathrm{~cm}$ du fond lagunaire, à partir de 48 transects Nord-Sud équidistant d'environ $1 \mathrm{~km}$ et sur quelques points isolés (Figure 2).

Pour l'identification des échantillons, les transects entamés par la berge Nord portent l'initial A tandis que ceux de la berge Sud sont affectés de l'initial B ; les sédiments sont ensuite marqués A/BT (Notransect) $S_{\text {(Noéchantillon). }}$ 


\subsection{Analyses}

L'analyse macroscopique et au toucher des sédiments a permis d'apprécier la couleur, la présence de coquilles et des débris végétaux et les faciès lithologiques.

$\mathrm{Au}$ laboratoire, les échantillons débarrassés des coquilles et débris végétaux ont été étuvés à $60{ }^{\circ} \mathrm{C}$ pour la déshydratation. Une masse de $200 \mathrm{~g}$ de chaque échantillon de sédiments riches en sables a été prélevé, puis passée à un tamisage humide sur un tamis à mailles carrées, d'arêtes égales à $50 \mu \mathrm{m}$.

Ce tamisage humide permet de séparer la fraction grossière (diamètres grains $>50 \mu \mathrm{m}$ ) de la fraction fine (diamètres des grains $<50 \mu \mathrm{m}$ ). La fraction grossière retenue est séchée à l'étuve, puis pesée sur une balance de précision au millième de gramme. Le refus ainsi séché et pesé, subit un tamisage à sec sur une colonne constituée des tamis à mailles carrées de la série AFNOR d'arêtes suivantes : $2 \mathrm{~mm} ; 1 \mathrm{~mm} ; 0,5 \mathrm{~mm} ; 0,25 \mathrm{~mm} ; 0,125 \mathrm{~mm} ; 0,063 \mathrm{~mm}$. Le tamisage est effectué par un appareil de type "Rotap" pendant 10 minutes avec une amplitude de 70 vibrations/minute. Ce temps de tamisage permet de réduire raisonnablement l'erreur relative au poids d'une fraction à moins de 2\% (Tricart et Cailleux, 1959). Chaque refus de tamis est pesé et la masse enregistrée à des fins statistiques. 


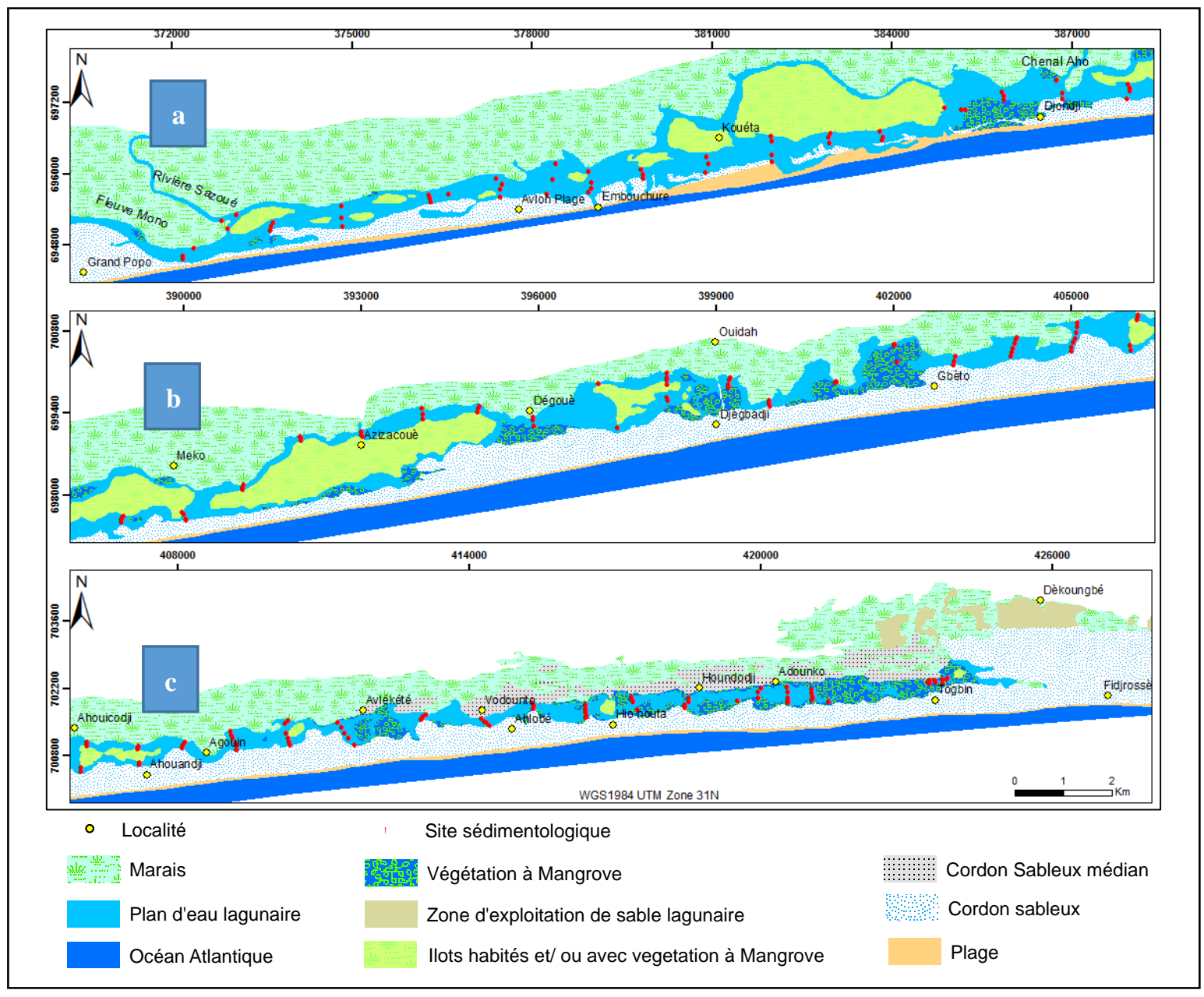

Fig. 2. Carte de répartition des points de prélèvement sédimentologique sur la lagune côtière découpée en 3 secteurs d'Ouest en Est :

a-) Partie Ouest : de la confluence du Mono et de la Sazoué au voisinage de Méko après

le chenal Aho ;

b-) Partie centrale : du village de Méko à Ahouicodji ;

c-) Partie Est : du village de Ahouicodji à Togbin.

\subsection{Traitements}

Afin de caractériser la couverture sédimentaire de la lagune côtière, les données de masse issues des analyses granulométriques ont servi à tracer des courbes de fréquences et principalement des courbes cumulatives à partir du logiciel Origin 6.0. L'échelle logarithmique définie par Krumbein et Pettijohn (1938), à travers la relation :

$$
\phi=-\log _{2}(\mathrm{~d})
$$

( $\mathrm{d}$ : étant le diamètre des grains en $\mathrm{mm}$ ) est considérée pour cette étude. 


\subsubsection{Indices granulométriques}

A partir, des courbes cumulatives, la médiane a été déterminée et les indices granulométriques calculés selon les formules de Folk \& Ward (1957). La médiane (Md) : représente la taille correspondant à $50 \%$ des grains déterminée directement sur la courbe cumulative.

La moyenne granulométrique (Mz): représente la distribution granulométrique moyenne des sédiments et permet l'individualisation d'un certain nombre de faciès. Il renseigne sur l'énergie cinétique moyenne lors du dépôt des sédiments. La formule est la suivante :

$$
M_{z}=\frac{\emptyset_{16}+\emptyset_{50}+\emptyset_{84}}{3}
$$

Tableau 1: Terminologie de caractérisation de la moyenne (Mz)

\begin{tabular}{|l|l|}
\hline $\mathrm{Mz}<1,00$ & Sables grossiers et graviers \\
\hline $1,00<\mathrm{Mz}<2,31$ & Sables moyens \\
\hline $2,31<\mathrm{Mz}<3,32$ & Sables fins \\
\hline $3,32<\mathrm{Mz}<4,00$ & Sables très fins \\
\hline $\mathrm{Mz}>4,00$ & Silts et argiles (lutites) \\
\hline
\end{tabular}

L'indice de tri ou de classement ou Sorting index $\left(\sigma_{i}\right)$ : renseigne sur l'hydrodynamisme lors du dépôt du sédiment. Il indique la qualité du classement ; plus il est faible, plus le sédiment est bien classé. Sa formule est:

$$
\sigma_{i}=\frac{\left(\emptyset_{84}-\emptyset_{16}\right)}{4}+\frac{\left(\varnothing_{95}-\emptyset_{5}\right)}{6,6}
$$

Tableau 2 : Terminologie de caractérisation du Sorting index $\left(\sigma_{i}\right)$

\begin{tabular}{|c|c|}
\hline $0<\sigma_{\mathrm{i}}<0,35$ & Très bien classé \\
\hline $0,35<\sigma_{\mathrm{i}}<0,5$ & Bien classé \\
\hline $0,50<\sigma_{\mathrm{i}}<0,7$ & Assez bien classé \\
\hline $0.7<\sigma_{\mathrm{i}}<1$ & Moyennement classé \\
\hline $1<\sigma_{\mathrm{i}}<2$ & Médiocrement classé \\
\hline $2<\sigma_{\mathrm{i}}<4$ & Très mal classé \\
\hline $4<\sigma_{\mathrm{i}}$ & Extrêmement mal classé \\
\hline
\end{tabular}

L'indice d'asymétrie ou Skewness ou (Sk) : traduit le degré d'asymétrie de la courbe de distribution par rapport à la médiane. Ce paramètre renseigne sur l'enrichissement en particules grossières (asymétrie négative), l'enrichissement en particules fines mal triées (asymétrie positive), ou s'il y a symétrie (Skewness compris entre -0.10 et 0.10 ). Sa formule est :

$$
\operatorname{Sk}=\frac{\emptyset_{16}+\emptyset_{84}-2 \emptyset_{50}}{2\left(\emptyset_{84}-\emptyset_{16}\right)}+\frac{\emptyset_{5}+\emptyset_{95}-2 \emptyset_{50}}{2\left(\varnothing_{95}-\emptyset_{5}\right)}
$$

Tableau 3 : Terminologie de caractérisation du Skewness (Sk)

\begin{tabular}{|l|l|}
\hline$+1,00>\mathrm{Sk}>+0,30$ & Très asymétrique vers les fins \\
\hline$+0,30>\mathrm{Sk}>+0,10$ & Asymétrique vers les fins \\
\hline$+0,10>\mathrm{Sk}>-0,10$ & Presque symétrique \\
\hline$-0,10>\mathrm{Sk}>-0,30$ & Asymétrique vers les grossiers \\
\hline
\end{tabular}


$-0,30>\mathrm{Sk}>-1,00$

Très asymétrique vers les grossiers

L'indice d'acuité ou Kurtosis $\left(\mathbf{K}_{\mathbf{G}}\right)$ : est l'indice d'acuité du mode ; il mesure l'angulosité de la courbe des fréquences. Si le mode est concentré dans des classes granulométriques restreinte, on a une distribution leptocurtique, s'il est dispersé on a une distribution platicurtique. Sa formule est :

$$
\mathrm{K}_{\mathrm{G}}=\frac{\emptyset_{95}-\emptyset_{5}}{2,44\left(\varnothing_{75}-\emptyset_{25}\right)}
$$

Tableau 4 : Terminologie de caractérisation du Kurtosis $\left(K_{G}\right)$

\begin{tabular}{|l|l|}
\hline $\mathrm{K}_{\mathrm{G}}<0,67$ & Courbe très platycurtique \\
\hline $0,67<\mathrm{K}_{\mathrm{G}}<0,90$ & Courbe platycurtique \\
\hline $0,90<\mathrm{K}_{\mathrm{G}}<1,11$ & Courbe mesocurtique \\
\hline $1,11<\mathrm{K}_{\mathrm{G}}<1,50$ & Courbe leptocurtique \\
\hline $1,50<\mathrm{K}_{\mathrm{G}}<3,00$ & Courbe très leptocurtique \\
\hline $3,00<\mathrm{K}_{\mathrm{G}}$ & Courbe extrêmement leptocurtique \\
\hline
\end{tabular}

\subsubsection{Mode de transport des sédiments}

La relation entre le calibre d'une particule et le mode de transport puis les conditions de dépôt est révélée par le diagramme de Passega (1964). Ce diagramme prend en ordonnée $(\mathrm{C})$, le premier percentile (dimension à laquelle 99\% du sédiment sont inférieurs dans le système de cumulation rétrograde), et en abscisse (M), la valeur de la médiane (en $\mu \mathrm{m})$ de la distribution granulométrique (Oyédé, 1983).

Les portions de l'enveloppe du diagramme de Passega distinguent :

- un transport par charriage (saltation ou roulement) représenté par les portions NO (sables grossiers); OP, PQ, QR (sables),

- un transport en suspension gradée : portion RS

- un transport en suspension uniforme T.

\subsubsection{Origine des sédiments}

Pour la détermination de l'origine des sédiments, les diagrammes KSk de Friedman (1962), Md-Sk, Mz-Sk de Moiola et Weiser (1968), (avec Md, $\mathrm{Mz}, \mathrm{K}, \mathrm{Sk}$ ) respectivement la médiane, la moyenne et les indices d'acuité, asymétrie), établissent une discrimination entre les sables de plages, de rivières, de dunes côtières et de dunes continentales (Boumir, 1985 ; Brahim et al., 2008 ; N'guessan et al., 2011).

\subsubsection{Carte de lithofaciès}

Afin de faire ressortir la répartition spatiale des faciès sédimentaires, une carte de lithofaciès a été réalisée via le logiciel ArcGIS 10.3. La classe des sables a été discriminée suivant la classification retenue par Udden (1914), Wentworth (1922) et Blott Pye (2001) (Tableau 4), auxquels s'ajoute la classe des vases sableuses et celles des vases. 
Tableau 4 : Discrimination de la classe des sables

\begin{tabular}{|c|c|c|}
\hline Taille de grain $\varnothing$ & Taille de grain $\mu \mathrm{m}$ & Terminologie \\
\hline 0 & 1000 & Sable très grossier \\
\hline 1 & 500 & Sable grossier \\
\hline 2 & 250 & Sable moyen \\
\hline 3 & 125 & Sable fin \\
\hline 4 & 63 & Sable très fin \\
\hline
\end{tabular}

\section{Résultats et discussion}

3.1. Analyse macroscopique et granulométrique des sédiments superficiels de la lagune côtière

La couverture sédimentaire de la lagune côtière est constituée de quatre classes de faciès lithologiques : sables, sables vaseux, vases sableuses, vases. Les faciès à galets et graviers n'ont pas été mis en évidence sur le fond lagunaire. Cette absence peut être liée au domaine pourvoyeur du matériel clastique, l'incidence du barrage de Nangbéto et la localisation de la lagune dans une plaine littorale à faible pente encombrée de marais. Selon Rossi et Antoine (1990), le lit mineur du fleuve Mono (principal tributaire de la lagune), contient exclusivement des sables et graviers dont les diamètres diminuent régulièrement de l'amont vers l'aval (Figure 3).

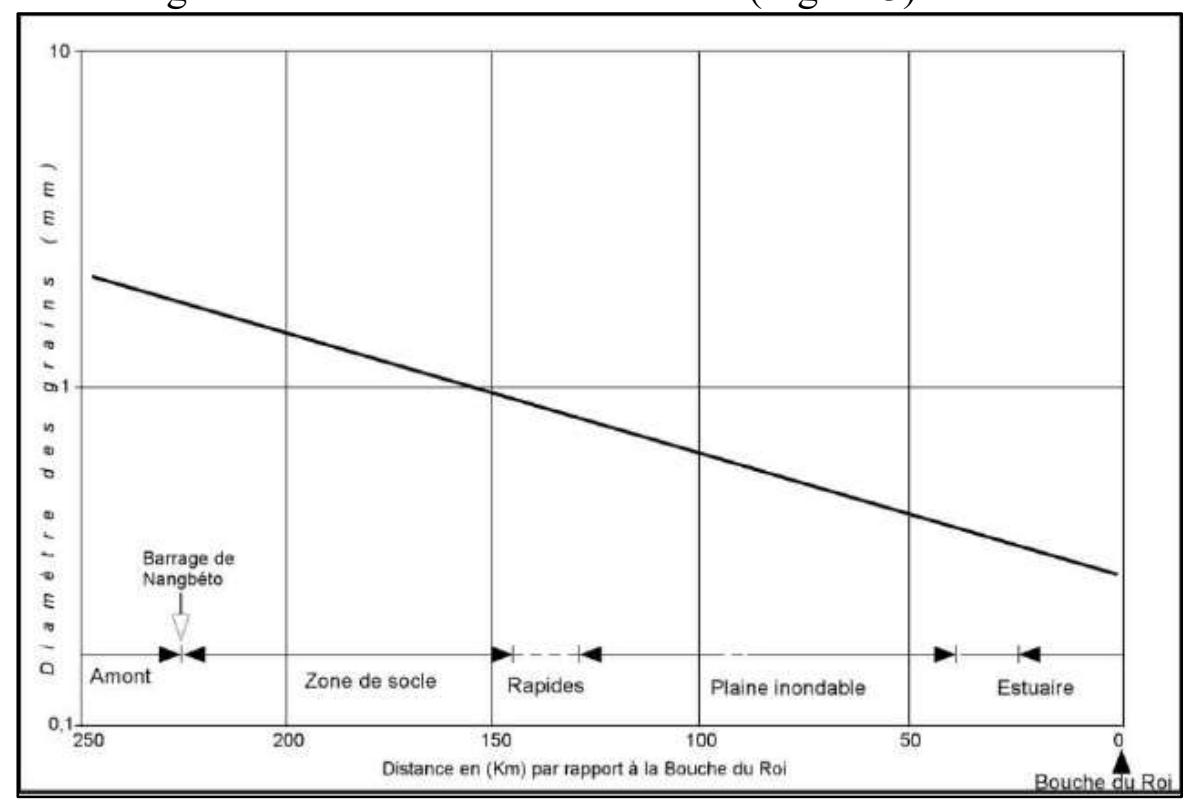

Fig. 3. Echelonnement des diamètres moyens de sables le long du fleuve Mono Rossi et Antoine (1990).

Les sables sont de couleur variable : brun, gris, jaune roux et parfois sombre. Les vases sont le plus souvent de couleur sombre et parfois verdâtre à vert-olive. La couleur des sédiments mixtes (vases sableuses et sables 
vaseux) découle du mélange des couleurs des deux faciès qui les composent. Les sédiments prélevés dans le bassin lagunaire contiennent généralement des débris végétaux et/ou coquilliers. La couleur des sables est à mettre en relation avec la présence des cordons de sables gris et brun qui encadrent la lagune sans occulter les sables de la plage qui peuvent rejoindre la lagune surtout dans le domaine des embouchures. La couleur sombre des vases pourrait être liée à la matière organique surtout dans les domaines de la végétation à mangrove.

\subsection{Les indices granulométriques \\ Moyenne granulométrique}

Les moyennes granulométriques des sables, malgré de rapides variations transversales comme longitudinales révèlent une grande importance de sables moyens dans les faciès sableux des échantillons de sédiments superficiels (Figure 4). Toutefois, quelques faciès de sables grossiers apparaissent en direction de l'embouchure et surtout dans la zone des anciennes embouchures déjà obstruées. L'analyse de cette distribution laisse entrevoir, à travers la tendance de la série, une diminution de la taille des grains de Grand-Popo vers Togbin. Une telle tendance permet de déduire que l'hydrodynamisme marqué par une vitesse de l'ordre de 0,78 à $1,4 \mathrm{~m} / \mathrm{s}$ vers le fond et un débit de $429 \mathrm{~m}^{3} / \mathrm{s}$, à la station de Avloh Plage (Ouest lagune) diminuerait progressivement en direction de Togbin (Est lagune).

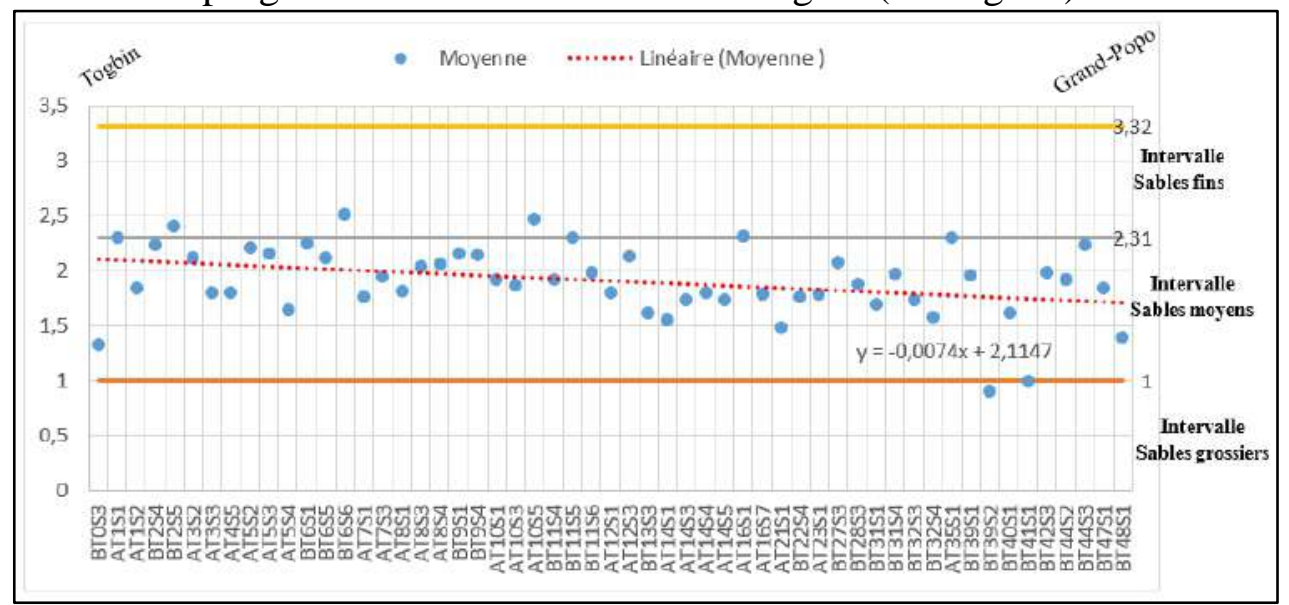

Fig. 4.Distribution de la moyenne granulométrique des sédiments à faciès sableux le long de la lagune côtière et tendance d'évolution.

\section{Indice de classement}

Les faciès sableux de la lagune côtière présentent généralement un classement médiocre avec quelques rares fois un classement moyen (Figure 5). Ces valeurs du classement sont caractéristiques des environnements de rivière et justifient ainsi les influences des cours d'eau et des ruissellements sur la dynamique sédimentaire du milieu lagunaire. Un tel classement 
médiocre peut être aussi lié à la présence de nombreux obstacles tels que les ilots et les parcs à poissons qui amenuisent l'hydrodynamisme et concentrent des particules de toutes tailles dans différents secteurs de la lagune.

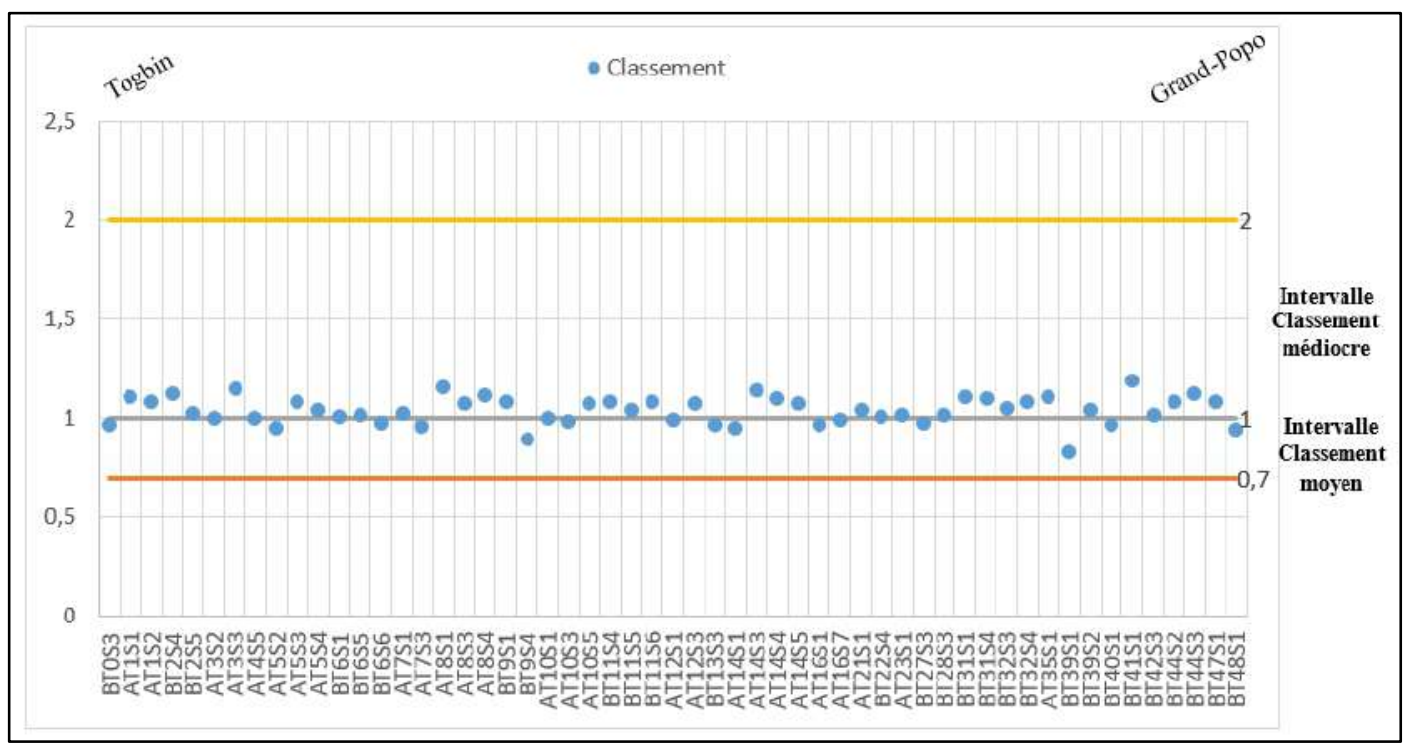

Fig. 5. Distribution de l'indice de classement des sédiments à faciès sableux le long de la

\section{Indice d'asymétrie} lagune côtière.

Les sédiments sableux de la lagune présentent un indice d'asymétrie qui tend de presque symétrique à une asymétrie vers les fins (Figure 6). Ce qui témoigne de l'influence de plusieurs sources d'apports sédimentaires avec des courants de différentes intensités mais généralement faibles dans ce milieu. En sus du fleuve Mono, la rivière Sazoué, le chenal Ahô, l'océan Atlantique, les eaux de ruissellement qui drainent le cordon sableux médian comme subactuel. Selon Pimmel (1984), les brusques changements de l'énergie et de la compétence du courant d'écoulement provoquent l'alternance de niveaux sableux et argileux donc, un mélange de sédiments fins, moyens et grossiers.

\section{Indice d'acuité}

L'indice d'acuité révèle que le mode de la plupart des fractions sableuses de la lagune n'est ni restreint, ni dispersé dans les classes granulométriques (Figure 7). Les échantillons présentent des courbes mésocurtiques. Mais, la courbe des sédiments prélevés dans la zone des anciennes embouchures et près de la plage présente des allures leptocurtiques. Les valeurs ainsi obtenues informent sur la qualité du classement et renseignent encore sur la multiplicité de différentes sources d'apports (plusieurs stocks granulométriques). Cet ensemble laisse entrevoir une variation hydrodynamique très contrastée et à partir de diverses sources 
d'approvisionnement. Une telle variation peut aussi trouver son origine dans les changements saisonniers du régime hydrologique des cours d'eau liés à la variabilité pluviométrique.

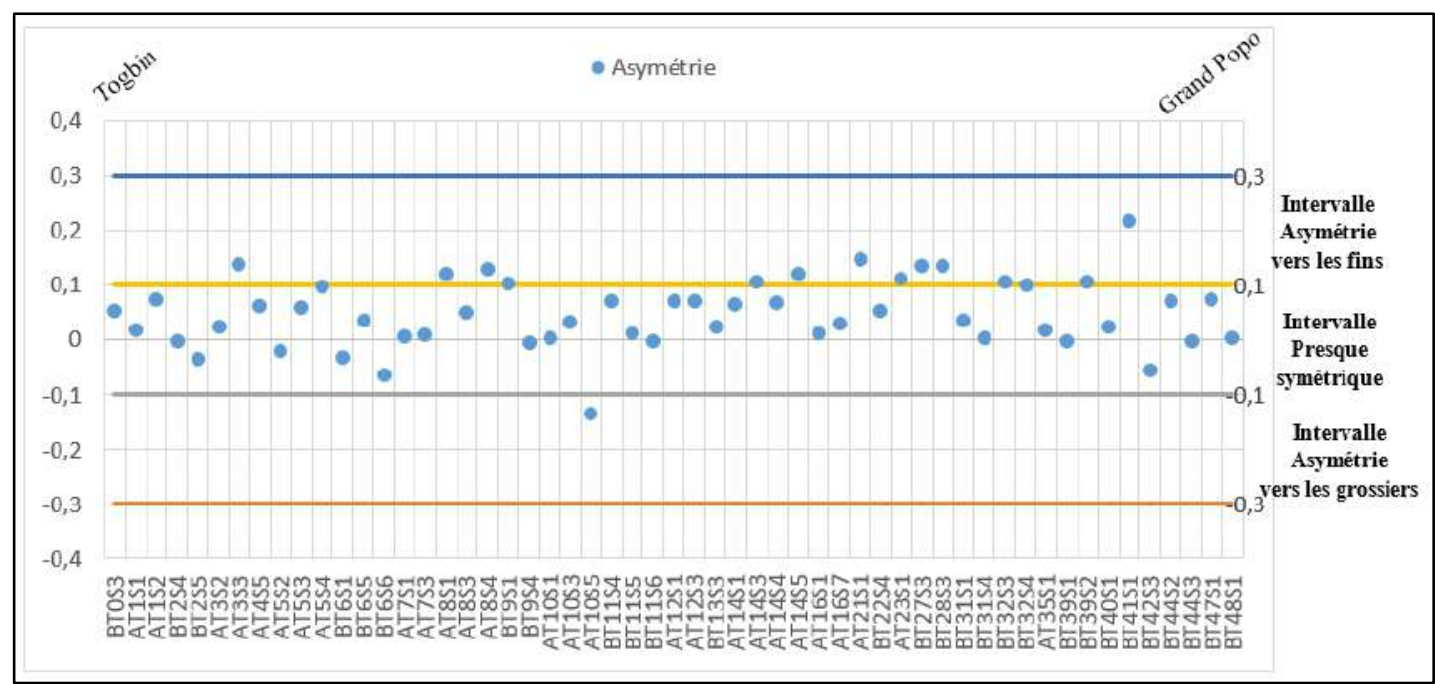

Fig. 6. Distribution de l'indice d'asymétrie des sédiments à faciès sableux le long de la lagune côtière.

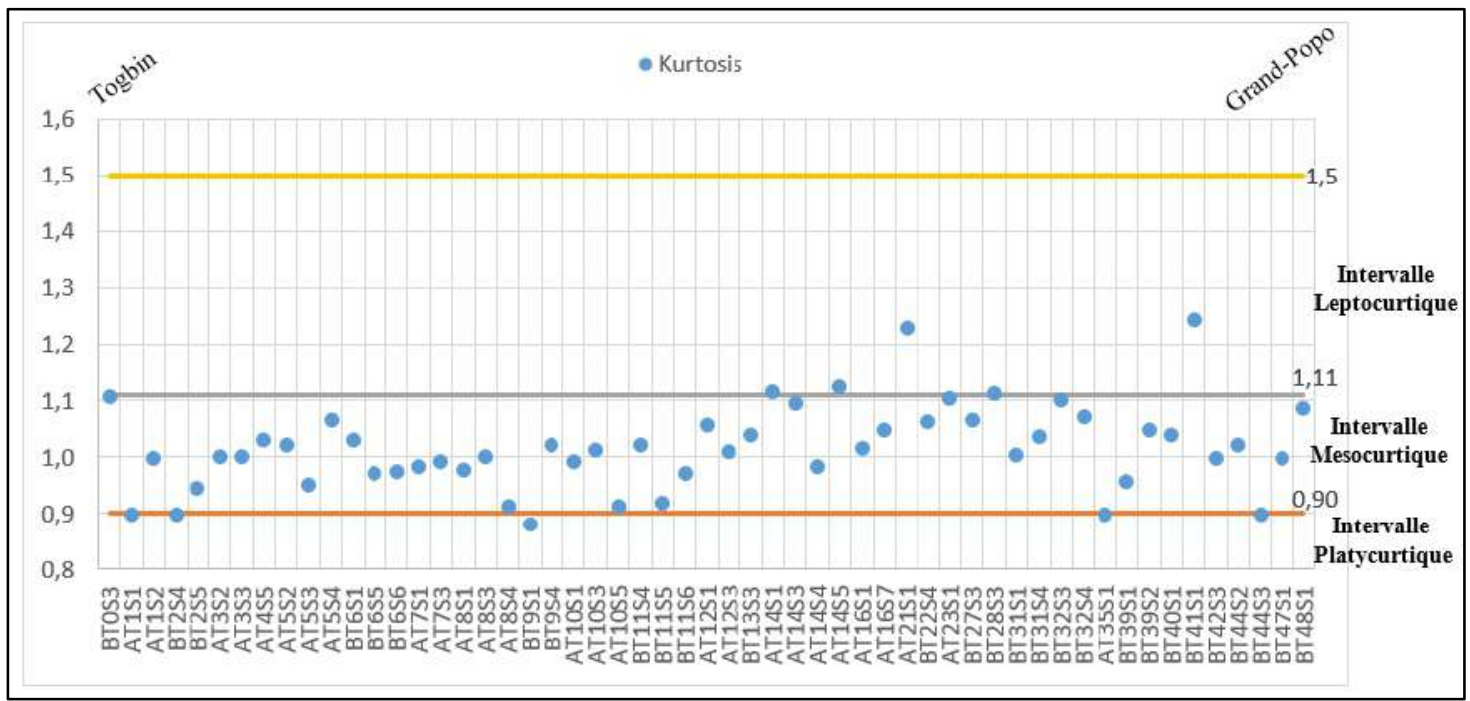

Fig. 7. Distribution de l'indice d'acuité des sédiments à faciès sableux le long de la lagune côtière.

\subsection{Interprétation des courbes granulométriques des sables de la lagune côtière}

Les courbes cumulatives semi-logarithmiques des sables étudiés présentent des allures quasi sigmoïde (en forme de $\mathrm{S}$ ) à faible pente, qui tendent vers une forme hyperbolique plus ou moins régulière (Figure 8). Elles sont caractéristiques des milieux lagunaires et des embouchures de cours d'eau 
(Rivière, 1977 ; Bouden et al., 2009). Selon Tricart (1965), le dépôt s'est fait suite à la variation de la compétence du courant de transport. Toujours dans le même sens, Rivière (1977) affirme que le faciès hyperbolique indique une réduction progressive de la force de l'agent de transport. Cet agent qui était initialement capable de transporter les grains d'une certaine dimension ne l'est plus, suite à une réduction de sa vitesse, donc de sa force de transport ; par conséquent, il abandonne les grains sur place. Ces analyses viennent renforcer les variations hydrodynamiques soulignées par les indices granulométriques.

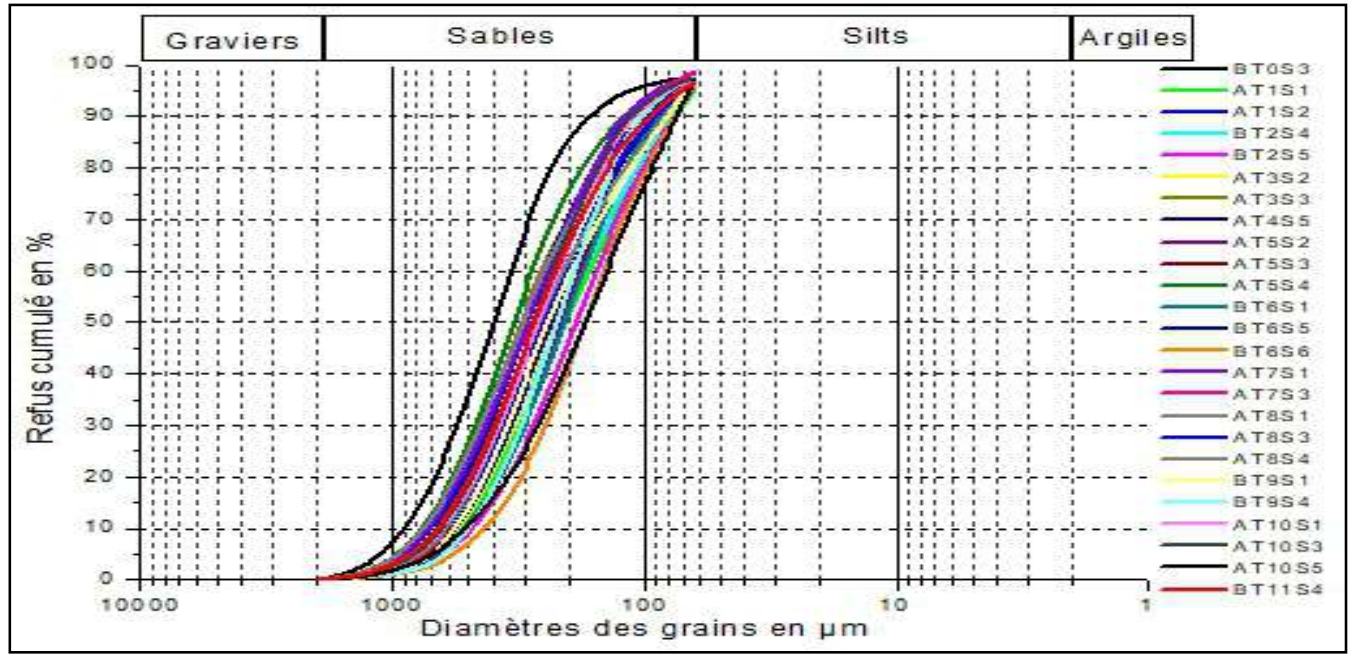

Fig. 8. Courbes granulométriques semi-logarithmiques_de quelques faciès sableux

\subsection{Mode de transport}

Même si la dispersion des points n'épouse pas exactement l'enveloppe de Passega (Figure 9), il permet néanmoins de faire ressortir que la quasitotalité des sables de la lagune occupe les portions OP, PQ et, dans une moindre mesure, la portion ON (sédiments dans le secteur des embouchures et du Mono côté plage). Ainsi, le transport de ces sables se fait généralement par saltation (PQ) et roulement avec suspension gradée (OP). 


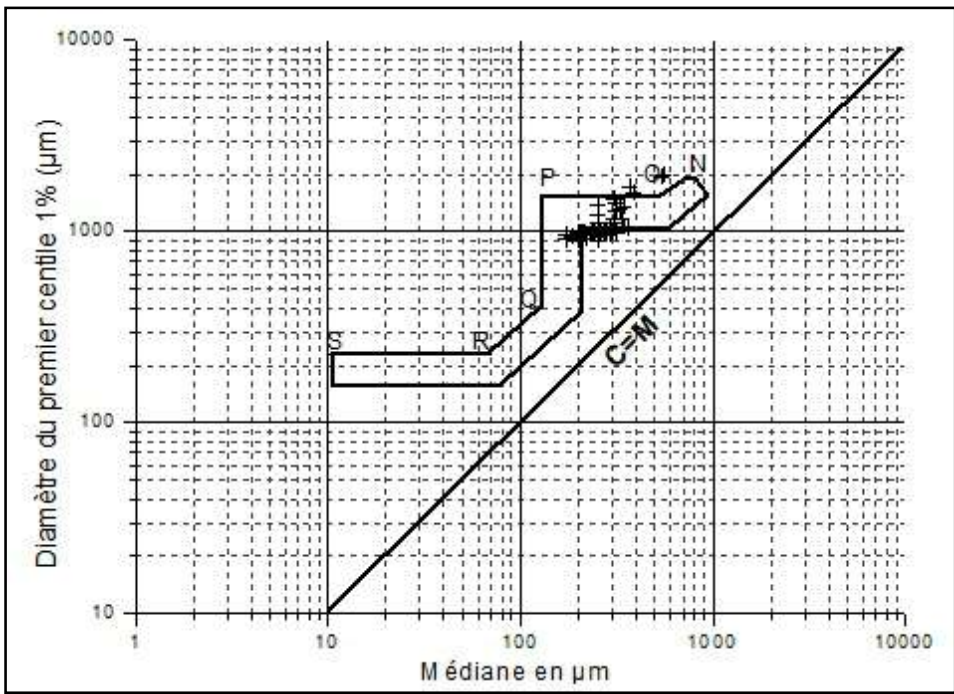

Fig. 9. Diagramme C/M de Passega pour les sédiments de la lagune côtière.

\subsection{Origine des sédiments}

L'analyse du diagramme K-Sk de Friedman (1962) (Figure 10) montre que les sables de lagune côtière sont apportés par les rivières (cours d'eau) avec aussi une origine dans le domaine de plage. Les diagrammes Md-Sk, MzSk de Moiola et Weiser (1968) (Figure 11 et 12), confirment les apports du domaine de plage avec une précision sur les apports du domaine des dunes côtières au détriment des dunes continentales.

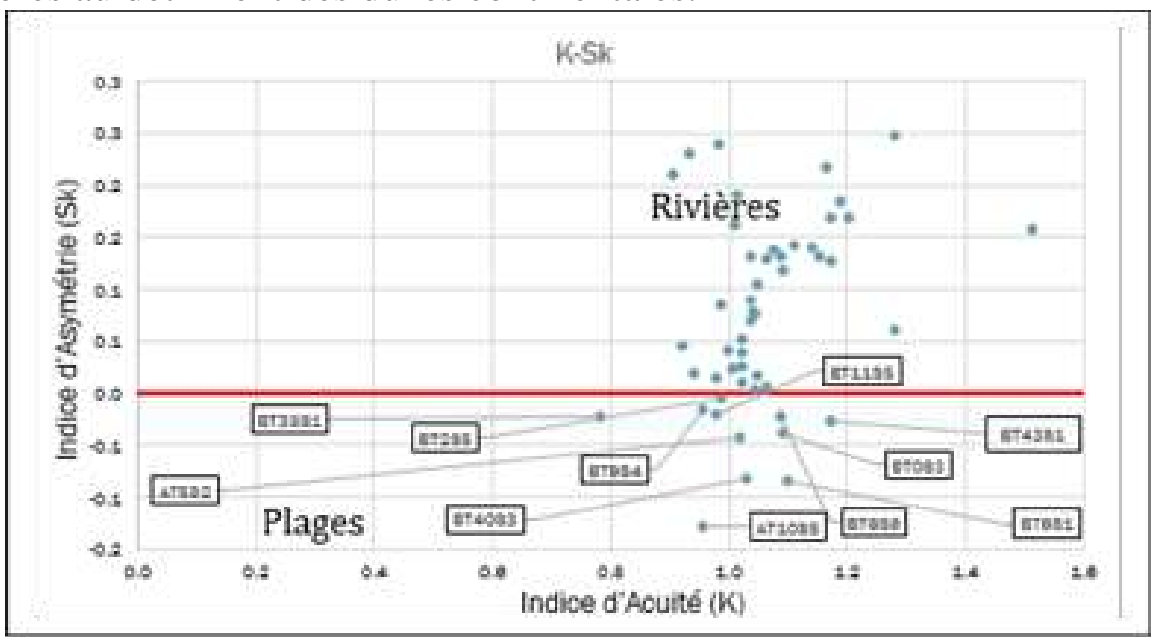

Fig. 10. Répartition K-Sk des sédiments de la lagune côtière 


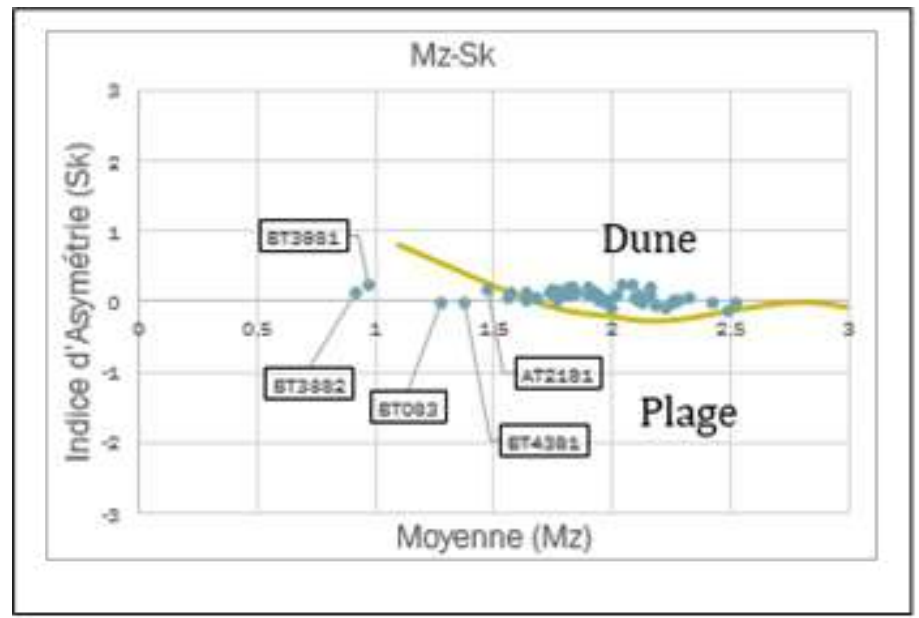

Fig. 11. Répartition Mz-Sk des sédiments de la lagune côtière

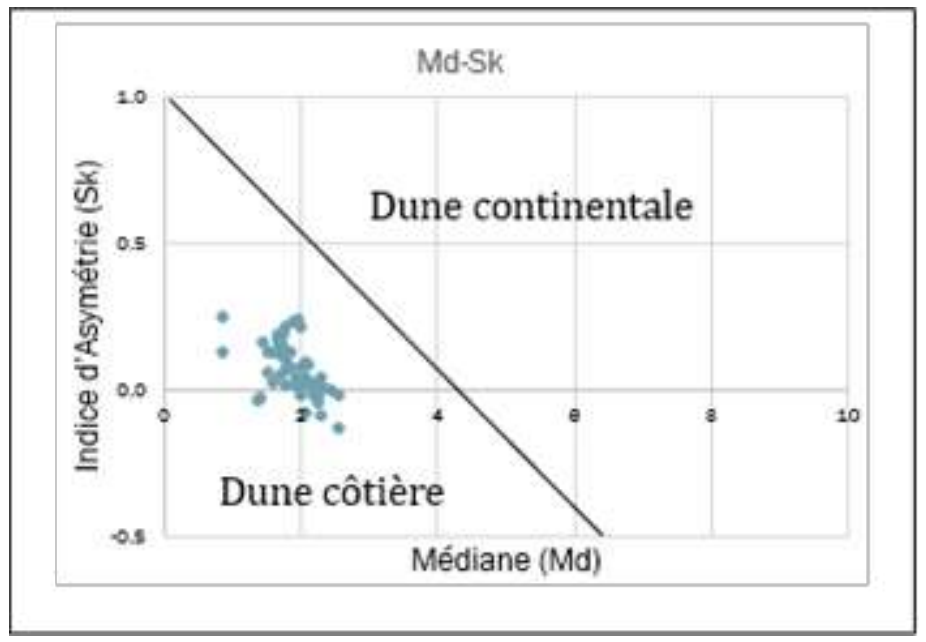

Fig. 12. Répartition Md-Sk des sédiments de la lagune côtière

\subsection{Répartition spatiale des faciès de la lagune côtière}

L'analyse de la carte de lithofaciès (Figure 13), permet de déduire l'absence d'un gradient de répartition concentrique des sédiments telle que décrite par Guelorget et Perthuisot (1983) et observée sur le "lac" Ahémé avec des vases au centre évoluant vers les sables sur les berges (Hounkpè, 2017). Cette carte de lithofaciès laisse plutôt entrevoir, dans la partie Ouest correspondant à la lagune de Grand-Popo, un gradient de répartition longitudinal avec les dépôts sableux du côté de l'océan et les dépôts de plus en plus fins vers la terre. Une telle organisation peut être aussi observée sur la lagune de Korba en Tunisie (Bouden et al., 2009). 
Dans le secteur Est correspondant à la lagune de Ouidah, la répartition des faciès sédimentaires est sous l'influence de plusieurs facteurs tels que : la présence des ilots, des multiples chenaux et sources d'apports, sans oublier les pratiques anthropiques. Cette distribution qualifiée d'intermédiaire ou sans gradient de répartition adéquat est observée sur le "lac" Nokoué (Texier et al., 1980).

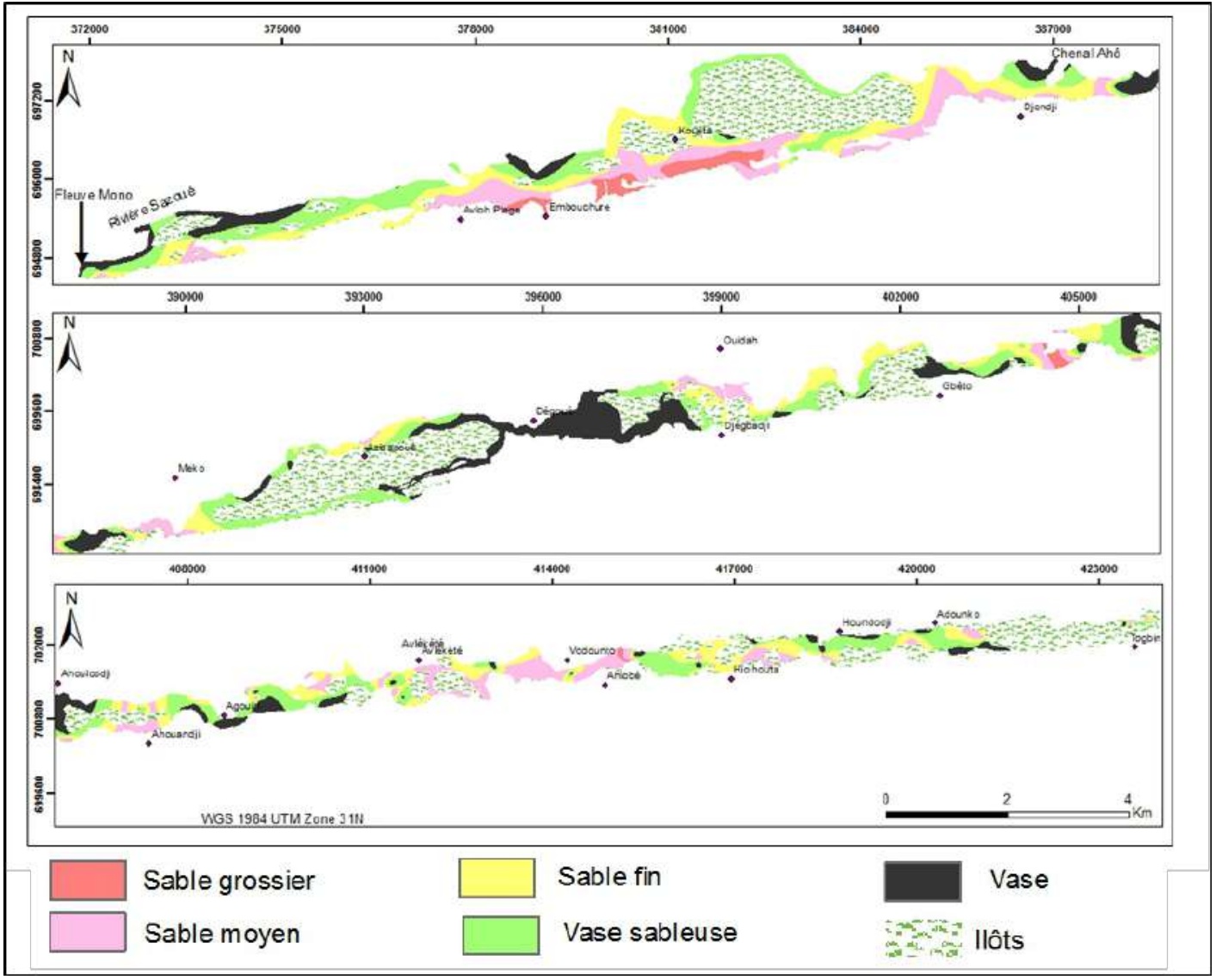

Fig. 13. Carte des lithofaciès de la lagune côtière.

En définitive, la lagune côtière est un plan d'eau situé entre deux cordons sableux qui sont des dunes côtières d'âges différents et largement décrites dans la littérature (Oyédé, 1991 ; Laïbi, 2011), comme les cordons médians de sable gris, au nord de la lagune et les cordons subactuels de sable brun de 1 à $4 \mathrm{~m}$ d'altitude. Le matériel sableux peut-être remobilisé vers la lagune de façon naturelle (ruissellement, vent, phase de débordement de la lagune) et/ou anthropique (aménagement des berges...). Ce plan d'eau est un prolongement du cours inférieur du fleuve Mono qui draine des sédiments depuis la région du socle. A cela s'ajoute le transfert du matériel bioclastique via la rivière Sazoué et le système Couffo-Ahémé par le truchement du chenal Ahô et l'entrée des eaux marines. Ces différentes sources d'alimentation 
hydrosédimentaire, la variation saisonnière de l'intensité des courants et les migrations de l'embouchure influencent la dynamique sédimentaire de la lagune et corroborent les résultats de la distribution des indices granulométriques et les différents diagrammes. De plus, la présence de plusieurs ilots, et des parcs à poissons réduisent l'hydrodynamisme de ce plan d'eau, surtout dans le secteur de la lagune de Ouidah, où ces obstacles sont légion et renforcent la tendance à l'envasement de ce plan d'eau.

\section{Conclusion}

L'étude des sédiments de la lagune côtière à travers une approche granulométrique a permis de mettre en évidence une couverture de type arénolutitique au fond de cet écosystème aquatique du Bénin. L'hydrodynamisme de la lagune de Grand-Popo en relation avec les cours d'eau et les entrées marines est plus important que celui du secteur Est correspondant à la lagune de Ouidah. Le transit du matériel sédimentaire des bassins versants, auxquels s'ajoutent ceux des cordons sableux et de l'océan Atlantique enrichissent la lagune en matériel détritique. La rupture de communication directe entre le complexe fluvio-lagunaire occidental et oriental engendre un endoréisme dans la partie Est de la lagune côtière. Ainsi, avec la présence des ilots, de la végétation à mangrove et des différents types de parcs à poissons (impacts anthropiques), l'hydrodynamisme s'amenuise, conduisant inévitablement à une tendance à la sédimentation. Dans un contexte de variabilité climatique et de littoralisation, un plan de gestion doit être mis en œuvre pour le suivi hydrosédimentaire de la lagune côtière afin de préserver ses innombrables atouts.

\section{References:}

1. Blott, S.J. et Pye, K. (2001). GRADISTAT: A grain size distribution and statistic package for the analysis of uncololidated sediments. Earth Surface Processes and Landforms 26, 1237-1248.

2. Bouden, S., Chaabani, F. et Abdeljaoued, S. (2009). Dynamique sédimentaire de la lagune de Korba (nord-est de la Tunisie). Quaternaire, 20, (2), 227-237.

3. Boumir, K. (1985). Les sables "fauves " de la région de Méknès : éléments d'analyse sédimentologique. Bulletin de l'institut scientifique, Rabat, ${ }^{\circ}$ 9, 107-116.

4. Brahim, M., Bejaoui, B. et Atoui, A. (2008). Etude de l'hydrodynamique sédimentaire de la lagune de Bizerte ; Bull.Inst.Natn.Scien.Mer de Salammbô, vol.35, 12p.

5. Castaings, J. (2008). Etat de l'art des connaissances du phénomène de comblement des milieux lagunaires. Mémoire de Master 2 Gestion du 
Littoral et des Mers, Université de Montpellier 2 - Cepralmar, Réseau de Suivi Lagunaire, $100 \mathrm{p}$.

6. Folk, R.L. and Ward, W.C. (1957). Brazos rivers bar: a study in the significance of grain size parameters. Journal of Sedimentary Petrology, Vol. 27, $\mathrm{n}^{\circ}$ 1, 325-354.

7. Friedman, G.M. (1962). Comparison of moment measures for sieving and thin-section data for sedimentary petrological studies. Jour. Sedim. Petrol. 32, 15 - 25 pp.

8. Guelorget, O. et Perthuisot, J.P. (1983). Le domaine paralique : expressions géologiques, biologiques et économiques du confinement. Travaux du laboratoire de géologie, Presses de l'ENS, Paris, 136.

9. Hounkpe, J.B. (2017). Approches géochimiques de la contamination en métaux lourds des eaux et des sédiments du lac Ahémé au SudBénin. Thèse de Doctorat Unique en Science de la Terre et de l'Univers, option Géosciences de l'Environnement, Université d'Abomey-Calavi, 203p.

10. Krumbein, W.C. and Pettijohn, F.J. (1938). Manual of Sedimentary Petrography. Appleton-Century-Crofts, New-York.

11. Laibi, R.A. (2011). Dynamique actuelle d'une embouchure fluviale estuarienne à flèche sableuse, la Bouche du Roi, Bénin, Golfe de Guinée: caractérisation hydrosédimentaire et géomorphologique. Thèse de Doctorat, Université d'Abomey-Calavi et Université du littoral Côte d'Opale, 302p.

12. Maanan, M. (2003). Etude sédimentologique du remplissage de la lagune de Sidi Moussa (côte atlantique marocaine). Caractérisations granulométrique, minéralogique et géochimique. Thèse de Doctorat, Université Chouaib Doukkali, Maroc, 176 p.

13. Moiola, R.J. and Weiser, D. (1968). Textural parameters: an evaluation. Journal of Sedimentary Petrology, ${ }^{\circ} 38,45-53$.

14. N'guessan, Y.A., Amani, E.M., Koffi, B. K. F., Adopo, L., Diangone, B.E., Monde, S. et Kouadio, A. (2014). Caractérisation morphologique et sédimentologique de l'embouchure du fleuve Sassandra (Côte d'Ivoire) . Afrique SCIENCE 10(2), 277 - 288.

15. N'guessan, Y.A., Wognin, V., Coulibaly, A., Monde, S., Wango, T.E. et Aka, K. (2011). Analyse granulométrique et environnement de dépôts des sables superficiels de la lagune Adjin (Côte d'Ivoire). Revue Paralia, Vol. 4, 6.1-6.14.

16. Oyédé, L.M. (1983). Un exemple de sédimentation biodétrique quaternaire dans le domaine margino-littoral en climat tropical humide : le lac Ahémé- (Bénin - Afrique de l'Ouest). Thèse de Doctorat de 3e cycle de Géologie sédimentaire. Université du Bénin et de Dijon, 171p. 
17. Passega, R. (1964). Grain size representation by C.M. patterns as a geological tool. Journal of Sedimentary Petrology, 34(4),830.

18. Pimmel, A. (1984). Contribution à l'étude sédimentologique des mangroves de Casamance (Sénégal) : étude granulométrique et exoscopique. Thèse de Doctorat de spécialité (3ème cycle) en géologie, Institut de Géographie, Université Louis Pasteur, Strasbourg, France, $132 \mathrm{p}$.

19. Rivière, A. (1977). Méthodes granulométriques : techniques et interprétation. Ed Masson, Paris, New York, Barcelone, Milan, 149p.

20. Rossi, G. et Antoine, P. (1990). Impacts hydrologiques et sédimentologiques d'un grand barrage: l'exemple de Nangbéto (Togo-Bénin). Revue de géomorphologie dynamique, Tome XXXIX. 63-77.

21. Texier, H. et Colleuil, B. (1980). Le lac Nokoué, environnement margino-littoral béninois, bathymétrie, lithofaciès, salinités, mollusques et peuplements végétaux. Bull. Inst, Géol Bassin d'Aquitaine. $n^{\circ} 28,115-136$.

22. Tricart, J. (1965). Principes et méthodes de la géomorphologie. Masson et Cie, Paris, 22p.

23. Tricart J. et Cailleux, A. (1959). Initiation à l'études des sables et des galets. CDU. Paris, 202p.

24. Udden, J.A. (1914). Mechanical composition of clastic sediments. Bulletin of the Geological Society of America 25, 655-744.

25. Wentworth, K.H. (1922). A scale of grade and class terms for clastic sediments. Journal of Geology 30, 377-392. 